\section{Endoscopic Diagnosis of Malignant Melanoma in the Gastric Cardia - Report of a Case without a Detectable Primary Lesion}

Although malignant melanoma involving the stomach is commonly mentioned in association with autopsies (1), endoscopic experience with this gastric malignancy is still limited, and only a few cases have been illustrated in the literature (2-4). This rare clinical condition is even rarer in Asians who are much less vulnerable to malignant melanoma than Caucasians.

A 59-year-old man was admitted on January 14, 1994 with complaints of postprandial epigastralgia and intermittent tarry stool for two months. Physical examination revealed a dark-skinned and emaciated elderly worker with pale conjunctiva. During the initial clinical impression of a bleeding peptic ulcer, he received an upper gastrointestinal (UGI) barium meal study which revealed a large polypoid mass located in the cardia portion of the stomach. The UGI endoscopic findings were as follows (Figure 1): A black-pigmented polypoid mass, about $4.5 \mathrm{~cm}$ in diameter, grew in the gastric cardia, and lateral infiltration, along the direction of the fundus, was also noted by the presence of several spots with increased pigmentation; and the tumor surface was covered with a membranelike, friable and necrotic gastric mucosa which may have been the origin of the hemorrhage. Direct endoscopic biopsy confirmed the diagnosis of malignant melanoma by demonstrating the presence of melanin-containing tumor cells (Figure 2). This patient is unusual because no primary focus was found despite a thorough investigation of each melanocyte-containing tissue and a whole body survey using all available diagnostic tools. He refused to receive surgical intervention which was considered as the only chance to improve quality of life and to prolong life expectancy (5). He was followed up at our oncological department and thereafter developed severe hiccough, progressive dysphagia, eachexia and bilateral lower-leg edema. Abdominal sonography done on July 16 showed a $4.6 \times 3.8 \times 3.5 \mathrm{~cm}^{3}$ metastatic mass over the upper pole of the left kidney, multiple hepatic metastases, lymphadenopathy over the perigastric and para-aortic area and minimal ascites. Feeding jejunostomy was then performed. However, the patient developed pneumonia and died of septic shock on August 3.

\author{
J. T. Liang', S. C. Yu', P. H. Lee', K. J. Chang', C. L. Fang', \\ W.J. Lin', S. M. Chuang ${ }^{2}$ \\ 'Department of Surgery \\ 2Department of Pathology \\ National Taiwan University Hospital, Taipei, Taiwan, \\ Republic of China
}

\section{References}

1. Das Gupta T, Brasfield R: Metastatic melanoma: a clinicopathological study. Cancer 1964; 17: 1323-1339.

2. Reintgen DS, Thompson W, Garbutt J et al.: Radiologic, endoscopic, and surgical considerations of melanoma metastatic to the gastrointestinal tract. Surgery 1984; $95: 635-639$.

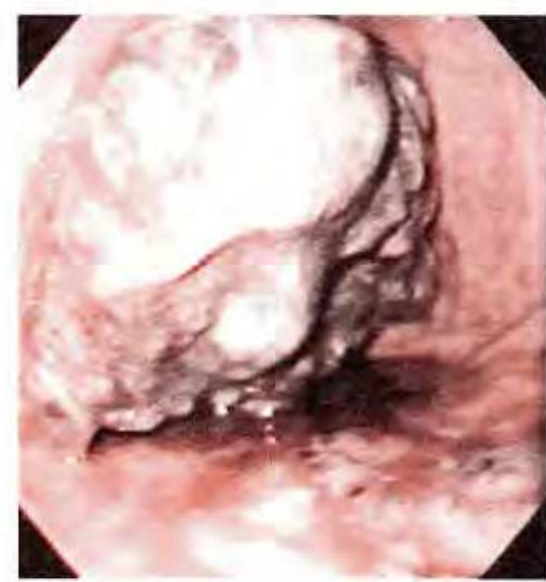

Figure 1: Endoscopic image of a large, black-pigmented, polypoid and superficially ulcerating mass in the gastric cardia. There are several spots of increased pigmentation lateral to the main mass.

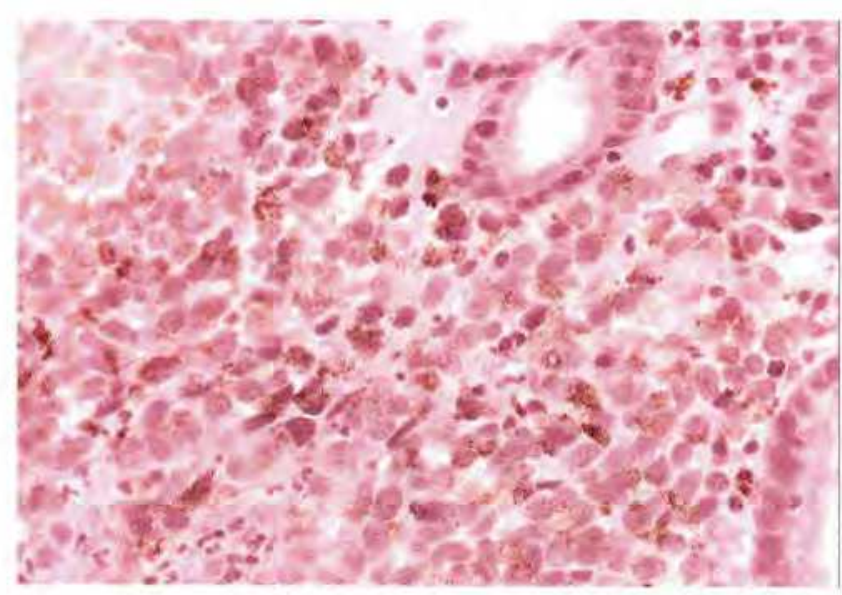

Figure 2: Endoscopic blopsy revealed that the gastric mucosa was infiltrated by numerous pleomorphic tumor cells with melanin deposits $\left(H \& E_{1} \times 400\right)$.

3. Bassi MSO, Colavolpe $V$ : Malignant melanoma metastatic to the stomach: endoscopic diagnosis and findings. Endoscopy 1980; 12: 86-89.

4. Nelson RS, Lanza F: Malignant melanoma metastatic to the upper gastrointestinal tract. Gastrointestinal Endoscopy 1978; 24: $156-158$.

5. Pector JC. Crokaert F, Lejeune F et al.: Prolonged survival after resection of a malignant melanoma metastatic to the stomach. Cancer 1988; 61: 2134-2135.

Corresponding Author

J. T. Liang, M.D.

Department of Surgery

National Taiwan University Hospital

No, 7, Chung-Shan South Road, Taipei, 10002

Taiwan, Republic of China 